\title{
Clinical implications of preoperative chemoradiotherapy prior to laparoscopic surgery for locally advanced low rectal cancer
}

\author{
KEISAKU KONDO ${ }^{1}$, TAIJU SHIMBO ${ }^{2}$, KEITARO TANAKA ${ }^{1}$, MASASHI YAMAMOTO ${ }^{1}$, \\ YOSHIFUMI NARUMI $^{2}$, JUNJI OKUDA ${ }^{3}$ and KAZUHISA UCHIYAMA ${ }^{1}$ \\ Departments of ${ }^{1}$ Gastroenterological Surgery and ${ }^{2}$ Radiology, Osaka Medical College; \\ ${ }^{3}$ Department of Surgery, Osaka Medical College Hospital Cancer Center, Takatsuki, Osaka 569-8686, Japan
}

Received May 19, 2016; Accepted November 3, 2016

DOI: $10.3892 / \operatorname{mco} .2016 .1098$

\begin{abstract}
The present study aimed to evaluate whether preoperative chemoradiotherapy (CRT) has any adverse effects on laparoscopic surgery (LS) for locally advanced low rectal cancer (LARC). The study was performed at the Osaka Medical College Hospital, and included patients who were operated on between July 2006 and December 2013. The short-term outcomes in 156 patients who underwent surgery for LARC following CRT were evaluated, of whom 152 underwent LS. Among the patients who were followed for $>40$ months, 77 patients (the CRT group) were compared with 39 patients who underwent LS without CRT (the surgery-alone group) for long-term outcomes. The total number of patients who received sphincter-preserving surgery was $74 \%$. No positive longitudinal resection margins were identified, and only $1.3 \%$ had identifiable positive circumferential resection margins. The complication rate was $14 \%$, and no serious complications occurred. There were no significant differences between the CRT and the surgery-alone groups in terms of the 5 -year relapse-free survival rate $(70.1$ vs. $61.5 \% ; \mathrm{P}=0.81)$ or the 5 -year overall survival rate $(88.3$ vs. $69.2 \%$; $\mathrm{P}=0.06)$. However, the 5-year local recurrence-free survival rate was significantly improved in the CRT group patients (96.1 vs. 79.5\%; $\mathrm{P}=0.009$ ). In conclusion, our results have demonstrated that LS with preoperative CRT appears to be feasible and safe, and may have beneficial effects on local recurrence.
\end{abstract}

\section{Introduction}

As the standard treatment for rectal cancer, open or laparoscopic total mesorectal excision (TME) with pre- or post-operative chemoradiotherapy (CRT) has resulted in a decrease in local

Correspondence to: Dr Keisaku Kondo, Department of Gastroenterological Surgery, Osaka Medical College, 2-7 Daigakumachi, Takatsuki, Osaka 569-8686, Japan

E-mail: sur086@osaka-med.ac.jp

Key words: low rectal cancer, preoperative chemoradiotherapy, laparoscopic surgery, short-term outcome, long-term outcome recurrence $(1,2)$. Although postoperative CRT for locally advanced rectal cancer markedly improves local control compared with surgery alone or surgery plus irradiation (3), preoperative CRT is considered to be the optimal therapeutic regimen for locally advanced low rectal cancer (LARC) due to its improved local control (4). Preoperative CRT may be associated with less acute toxicity and greater rates of sphincter-saving procedures, and may increase the probability of curative tumor resection when compared with postoperative CRT (4). Furthermore, tumor down-staging by CRT may lead to a complete clinical or pathological response (4). However, the postoperative complication rate of TME may increase with preoperative irradiation.

Although laparoscopic surgery is considered to be the best option available for the surgical treatment of rectal cancer due to its rates of local recurrence and survival, similar to those of open surgery, few reports in the literature have addressed the effects of preoperative CRT on laparoscopic surgery (5). The aim of the present study was to determine whether preoperative CRT has any adverse effects on laparoscopic surgery in patients with LARC.

\section{Materials and methods}

Ethical approval. The institutional ethics committee of Osaka Medical College Hospital approved the present study. Informed consent for the therapy was obtained from all the patients prior to chemoradiotherapy, after they had received a detailed description of the procedure and its likely complications.

Patients. This retrospective cohort study comprised 156 consecutive patients with histologically confirmed primary adenocarcinoma of the lower rectum with a distal margin of $<10 \mathrm{~cm}$ from the anal verge, who underwent laparoscopic or open surgical treatment following preoperative CRT at Osaka Medical College Hospital between July 2006 and December 2013. The indications for laparoscopic surgery at our hospital included a maximal tumor size not in excess of $10 \mathrm{~cm}$, and no evidence of either synchronous resectable liver metastasis or distant metastasis. Indications for preoperative CRT included full-thickness rectal cancers (T3 or T4), as staged by magnetic resonance imaging or multi-detector computed tomography, no prior radiation therapy administered to the pelvis, and no 
evidence of para-aortic lymph node metastasis. All patients were treated with 5-fluorouracil-based chemoradiation, at a 4,000 centrigray (cGy) total dose of pelvic irradiation. A daily fraction of 200 cGy was administered five times per week. Chemotherapy consisted of oral tegafur/uracil and leucovorin calcium. The dose of tegafur/uracil was $300 \mathrm{mg} / \mathrm{m}^{2}$, and that of leucovorin calcium was $75 \mathrm{mg} /$ day during the radiotherapy. The patients subsequently underwent surgery 6-8 weeks after having completed the CRT. Pathological staging of the cancers was performed according to postoperative pathological reports using the Japanese Classification of Colorectal Carcinoma, Second English Edition (6). The characteristics of the 156 patients (107 men and 49 women) are shown in Table I. The median age of the patients was 62 years (range, 35-80), and the median tumor size was $4.0 \mathrm{~cm}$ (range, $2-10 \mathrm{~cm}$ ). The distance from the anal verge was $0-5.0$ and $5.1-10.0 \mathrm{~cm}$ in 52.5 and $47.5 \%$ of the patients, respectively. Clinical stage T3 tumors were present in $96.8 \%$ of the patients, whereas $3.2 \%$ patients had T4 tumors.

In the present study, long-term outcomes were examined by comparing 77 of the 156 patients, who were followed for $>40$ months (the CRT group), with 39 patients who had undergone laparoscopic surgery without preoperative chemoradiotherapy for LARC between January 2004 and November 2008, and who were followed for $>60$ months (the surgery-alone group). Data on the surgery-alone group of patients was obtained retrospectively. The characteristics of the two groups are summarized in Table II. The mean age of the patients in the CRT group was $62.05 \pm 10.23$ years, and in the surgery-alone group, it was $62.46 \pm 9.18$ years $(\mathrm{P}=0.83)$. Of the patients, 49 in the CRT group were men and 28 were women, whereas in the surgery-alone group, 27 were men and 12 were women $(\mathrm{P}=0.64)$. The mean tumor size was $4.02 \pm 0.14 \mathrm{~cm}$ in the CRT group and $4.02 \pm 0.20 \mathrm{~cm}(\mathrm{P}=1.00)$ in the surgery-alone group. The distance from the anal verge was $4.13 \pm 2.03 \mathrm{~cm}$ in the CRT group, and $5.61 \pm 2.45 \mathrm{~cm}(\mathrm{P}<0.008)$ in the surgery-alone group. Of the tumors in the CRT group, 26 were well-differentiated tubular adenocarcinoma (tub1) and 49 were moderately differentiated tubular adenocarcinoma (tub2), whereas two were of other types. In the surgery-alone group, 22 of the tumors were tub1, and 17 were tub2 $(\mathrm{P}=0.09)$. In the CRT group, 13 patients had stage II, 42 had stage IIIa, and 22 had stage IIIb disease clinically, whereas in the surgery-alone group, 11 patients had stage II, 20 had stage IIIa, and 8 had stage IIIb disease clinically $(\mathrm{P}=0.32)$.

Surgical procedure. The five-port technique was used for laparoscopic surgery, which featured: A $12-\mathrm{mm}$ port at the navel; three 5-mm ports, one each in the upper-right and -left and lower-left abdominal quadrants; and a 12-mm port in the right lower quadrant, as shown in Fig. 1. An Endopath ${ }^{\mathrm{TM}}$ Probe Plus II spatula probe (Ethicon Endo-Surgery, Cincinnati, $\mathrm{OH}$, USA) was used for precise dissection. After proximal lymph-node dissection, TME of the rectum down to the floor of the pelvis was performed. Subsequently, while preserving the hypogastric nerve, dorsal dissection in the avascular plane between the mesorectum and the parietal pelvic fascia down to the pelvic floor was precisely and adequately performed. Care was taken not to damage the pelvic splanchnic nerve during dorsal dissection. Next, lateral dissection was completed, while ensuring that the hypogastric plexus was preserved.
Table I. Patient characteristics.

Characteristics

Number of patients

Age in years (median/range)

$62(35-80)$

Gender

Male

$107(68.6 \%)$

Female

Tumor size in $\mathrm{cm}$ (median/range)

$4.0(2-10)$

Tumor histology

Tub1/tub2

$151(96.8 \%)$

Poor/muc/sig

$5(3.2 \%)$

Depth of invasion

T3

$151(96.8 \%)$

$\mathrm{T} 4$

Tumor location

$0-5.0 \mathrm{~cm}^{\mathrm{a}}$

$82(52.5 \%)$

$5.1-10.0 \mathrm{~cm}^{\mathrm{a}}$

$74(47.5 \%)$

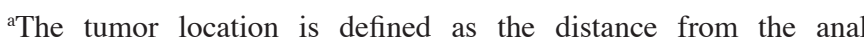
verge. Tub1, well-differentiated tubular adenocarcinoma; tub2, moderately-differentiated tubular adenocarcinoma; poor, poorly-differentiated adenocarcinoma; muc, mucinous adenocarcinoma; sig, Signet-ring cell carcinoma; T3, tumor invades through the muscular propria into the subserosa or into peritonealized pericolic or perirectal tissues; T4, tumor directly invades other organs or structures and/or invades the visceral peritoneum.

Dissection was performed up to the endopelvic fascia and levator ani muscle, taking great care to preserve the neurovascular bundle during the anterolateral dissection. Thus, in principle, autonomic nerve-preserving surgery was performed, except in the case of patients in whom we suspected direct tumor invasion of the neural plexus. Echelon60 ${ }^{\mathrm{TM}}$ (Ethicon Endo-Surgery) was used for rectal resection in low or super-low (anastomosis within $2 \mathrm{~cm}$ from the dentate line) laparoscopic anterior resection. The area of the rectum contralateral to the planned dissection site was retracted, and the tissue was pinched, as necessary, with the stapler to accomplish rectal transection using a single-fire staple cartridge. Stapling was performed from the anterior to the posterior rectum wall. The specimen was extracted through the diverting stoma incision, which was extended to $\sim 3-4 \mathrm{~cm}$, and the anastomosis was intracorporeally completed using the double-stapling technique. In cases of intersphincteric resection, the specimen was extracted via the anus and a hand-sewn colo-anal anastomosis was created. After abdominoperineal resection had been performed, the specimen was retrieved in the usual manner through a perineal incision. Primary perineal wound closure was performed, and a terminal colostomy in the left lower quadrant site was constructed. A well-experienced, board-certified laparoscopic colorectal surgeon (J.O.) supervised all the surgical operations.

Comparison between CRT and surgery-alone groups. The 5-year relapse-free survival rates (RFS), local pelvic recurrence free survival (LRFS) and 5-year overall survival rates (OS) were determined in 77 of the CRT group patients who were 
Table II. Comparison of patient characteristics in the two groups.

\begin{tabular}{lccr}
\hline Characteristics & Preoperative CRT group (n=77) & Surgery-alone group (n=39) & P-value \\
\hline Age (years) & $62.05 \pm 10.23$ & $62.46 \pm 9.18$ & 0.83 \\
Gender (male/female) & $72 / 27$ & $27 / 12$ & 0.64 \\
Tumor size (cm) & $4.02 \pm 0.14$ & $4.02 \pm 0.20$ & 1.00 \\
Tumor distance from the anal verge (cm) & $4.13 \pm 2.03$ & $5.61 \pm 2.45$ & $<0.01$ \\
Tumor histology (tub1/tub2/others) & $26 / 49 / 2$ & $22 / 17 / 0$ & 0.09 \\
cStage (II/IIIa/IIIb) & $13 / 42 / 22$ & $11 / 20 / 8$ & 0.32
\end{tabular}

Tub1, well-differentiated tubular adenocarcinoma; tub2, moderately-differentiated tubular adenocarcinoma; cStage, clinical stage.



Figure 1. Placement of the five laparoscopy ports. For stapler insertion, placement of the lower-right quadrant port is essential for successful transection of the rectum. The stapler should be inserted as caudally as possible. The numbers in the Figure indicate the size of the ports (in $\mathrm{mm}$ ).

followed for $>40$ months, and the rates were compared with those of the surgery-alone group.

Statistical analysis. Statistical analysis was performed using JMP 9 for Windows (SAS Institute, Inc., Cary, NC, USA). Correlations between categorical variables were assessed using the Chi-square test, and continuous data were evaluated using the Mann-Whitney U-test. Patient survival rates were calculated using the Kaplan-Meier method, and survival curves were compared using the log-rank test. $\mathrm{P}<0.05$ was considered to indicate a statistically significant difference.

\section{Results}

Surgical treatment and pathological data. The surgical data are summarized in Table III. The rate for low or super-low anterior resection was $64.7 \%$; that for intersphincteric resection was $15.4 \%$, and that for abdominoperineal resection was $17.3 \%$. Diverting ileostomies were created in all patients, with the exception of those who underwent abdominoperineal resection. Sphincter-preserving surgery was performed in $74 \%$ of the patients, and laparoscopic surgery was performed in $97.4 \%$ of the patients. The histological data following surgery are summarized in Table IV. None of the patients had a positive longitudinal resection margin, and only $1.3 \%$ of the patients had a positive circumferential resection margin.

The results of histological assessment of the responses to CRT are summarized in Table V. We evaluated responses using the Japanese Classification of Colorectal Carcinoma, Second English Edition (6). Accordingly, a grade 0 response (no effect) indicated that tumor cell necrosis or degeneration was not present in response to treatment. Grade 1a (minimal effect) indicated the presence of tumor cell necrosis or degeneration in less than one-third of the lesion. A grade $1 \mathrm{~b}$ response (mild effect) indicated the presence of tumor cell necrosis, degeneration and/or lytic changes in more than one-third, but less than two-thirds, of the lesion. A grade 2 response (moderate effect) indicated that prominent tumor cell necrosis, lytic changes, degeneration, and/or cancer disappearance occurred in more than two-thirds of the lesion, although viable tumor cells still remained. A grade 3 response (marked effect) indicated the presence of necrosis and/or lytic changes throughout the lesion, which were replaced by fibrosis with or without granulomatous changes, and no viable tumor cells were observed. Our results revealed that a grade 0 response was not observed in any patients; a grade 1a response was present in $31.4 \%$ of the patients; a grade $1 \mathrm{~b}$ response was present in $20.5 \%$ of the patients; a grade 2 response was in $29.5 \%$ of the patients; and a grade 3 response was in $18.6 \%$ of the patients. The resected specimen following surgery was submitted for pathological evaluation. Microscopic positive circumferential resection margins were present in only two cases, and microscopic positive distal resection margin cases were not observed in any of the cases (Table IV).

The surgical outcomes in the 156 patients who underwent CRT are summarized in Table VI. The total percentage of patients who experienced complications was $14.1 \%$; that of anastomotic leakage was $2.6 \%$, and that of wound infections was $5.8 \%$. All the identified infections were perineal wound infections following abdominoperineal resection. Three patients had ileus, and two patients required reoperation. Serious complications were not observed in the present study, and no patients succumbed to mortality in the hospital.

OS, RFS and LRFS. The median follow-up period in the CRT group patients was 58 months, and that in the surgery-alone group was 60 months. No significant differences were 
Table III. Surgical data.

\begin{tabular}{lc}
\hline Operative procedure & Number of patients (\%) \\
\hline Laparoscopic surgery & $152(97.4)$ \\
Low anterior resection/super-low & $101(64.7)$ \\
anterior resection & \\
Intersphincteric resection & $24(15.4)$ \\
Abdominoperineal resection & $27(17.3)$ \\
Open surgery & $4(2.6)$ \\
Abdominoperineal resection & $3(1.9)$ \\
Total pelvic exenteration & $1(0.6)$ \\
\hline
\end{tabular}

Table IV. Histological data following surgery.

\begin{tabular}{lc}
\hline Type of surgery & Number of patients $(\%)$ \\
\hline Circumferential resection margin & $2(1.3)$ \\
Positive & $154(98.7)$ \\
Negative & \\
Distal resection margin & $0(0)$ \\
Positive & $156(100)$ \\
Negative & \\
\hline
\end{tabular}

Table V. Histological assessments of response to preoperative chemoradiotherapy.

\begin{tabular}{lc}
\hline Response to NACRT & Number of patients $(\%)$ \\
\hline Grade & \\
0 & $0(0)$ \\
$1 \mathrm{a}$ & $49(31.4)$ \\
$1 \mathrm{~b}$ & $32(20.5)$ \\
2 & $46(29.5)$ \\
3 & $29(18.6)$
\end{tabular}

Histological criteria for assessment of response to neoadjuvant therapy were based on the Japanese classification of Colorectal Carcinoma, Second English Edition (6). NACRT, neoadjuvant chemoradiotherapy.

identified between the CRT group and the surgery-alone group in terms of the 5-year RFS (70.1 vs. $61.5 \%$; $\mathrm{P}=0.81$ ) (Fig. 2) and OS (88.3 vs. 69.2\%; P=0.06) (Fig. 3). The CRT group had a significantly higher rate of LRFS at 5 years compared with the surgery-alone group (96.1 vs. $79.5 \%$; $\mathrm{P}=0.009$ ) (Fig. 4). The rates of recurrence and/or metastasis following surgery are summarized in Table VII. Recurrence and/or metastasis occurred in $29.9 \%$ of the patients in the CRT group. Of these, lung metastasis accounted for $16.9 \%$, liver metastasis for $2.6 \%$, lymph node metastasis for $3.9 \%$, and local recurrence for $5.2 \%$. of the patients. By contrast, the recurrence and/or metastasis rate in the surgery-alone group
Table VI. Postoperative mortality and morbidity.

\begin{tabular}{lc}
\hline Characteristic & Number of patients $(\%)$ \\
\hline Postoperative complication & $22(14.1)$ \\
Anastomotic leakage & $4(2.6)$ \\
Wound infection & $9(5.8)$ \\
Ileus & $3(1.9)$ \\
Pelvic abscess & $3(1.9)$ \\
Urinary disorder & $3(1.9)$ \\
Mortality & $0(0)$
\end{tabular}

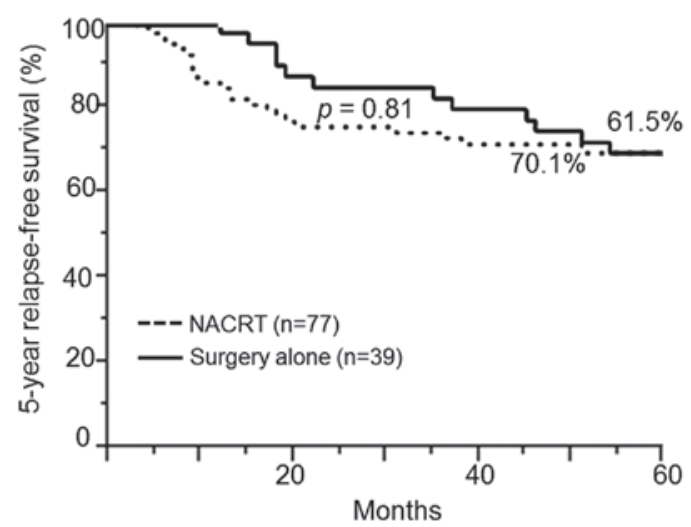

Figure 2. Five-year relapse-free survival rates in the two groups over the follow-up period. No significant differences were observed in the survival rates between the two groups. NACRT, neoadjuvant chemoradiotherapy.

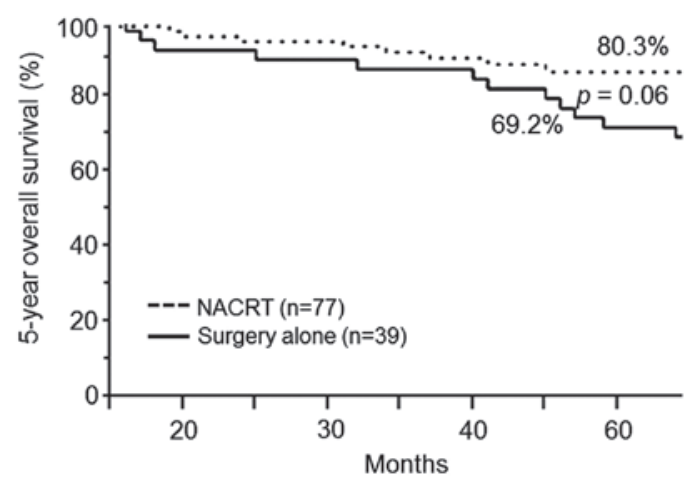

Figure 3. Five-year overall survival rates in the two groups. Survival rates between the two groups were not significantly different. NACRT, neoadjuvant chemoradiotherapy.

was $38.5 \%$, with local recurrence rates of $20.5 \%$, which was a higher percentage compared with that observed in the CRT group. Lung metastasis occurred in $7.7 \%$, and liver metastasis in $2.6 \%$, of the patients in the surgery-alone group. The rate of local recurrence was higher in the surgery-alone group compared with in the CRT group, whereas the CRT group had a higher rate of lung metastasis.

\section{Discussion}

The present study has suggested that laparoscopic surgery performed following preoperative CRT for advanced low 
Table VII. First recurrence and/or metastasis following surgery.

\begin{tabular}{lcr}
\hline Characteristic & Preoperative CRT group $(\mathrm{n}=77)(\%)$ & Surgeryalone group \\
\hline Presence of recurrence/metastasis & $23(29.9)$ & $15(38.5)$ \\
Local recurrence & $4(5.2)$ & $8(20.5)$ \\
Lung metastasis & $13(16.9)$ & $3(7.7)$ \\
Liver metastasis & $2(2.6)$ & $1(2.6)$ \\
Lung and liver metastasis & $1(1.3)$ & $0(0)$ \\
Lymph node metastasis & $3(3.9)$ & $1(2.6)$ \\
Others & $0(0)$ & $2(5.1)$
\end{tabular}

CRT, chemoradiotherapy.

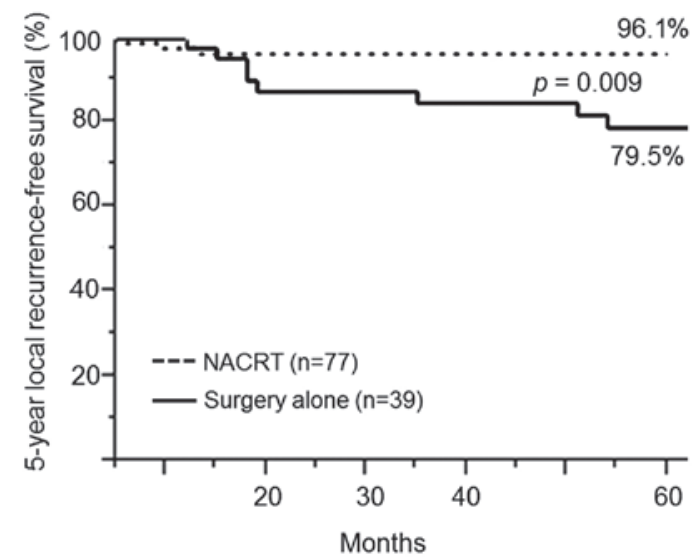

Figure 4. Five-year local recurrence-free survival rates in the two groups. Local recurrence-free survival rates in the preoperative chemoradiotherapy group was an improvement compared with that in the surgery-alone group. NACRT, neoadjuvant chemoradiotherapy.

rectal cancer may be safely performed by skilled surgeons. Laparoscopic resection of the colon is accepted as a method of surgical treatment for colonic cancer $(7,8)$. However, the laparoscopic procedure for rectal cancer is technically more difficult compared with that for colon cancer. Although a previous study has suggested the safety and feasibility of laparoscopic surgery for rectal cancer (9), its use continues to be controversial. However, laparoscopic surgery for colorectal cancers has been used to positive effect in our hospital (10-13) due to its advantages in providing a good view, even in narrow pelvises, and its more precise preservation of autonomic nerve preservation (14). The difficulty with surgery following CRT is partly explained by tissue inflammation, and the edema that may occur following CRT. Furthermore, although preoperative CRT in certain cases may blur the dissection plane due to fibrosis, it was possible to successfully perform nerve-preserving TME in all of our CRT laparoscopic cases, thereby suggesting the safety and feasibility of laparoscopic surgery following CRT.

The overall rate of postoperative complications in the preoperative CRT group was $14.1 \%$, of which only two patients required reoperation. These two male patients had undergone laparoscopic abdominoperineal resection (APR), and an ileus occurred due to prolapse of the small intestine to the pelvic floor. At present, since the current procedure is that the pelvic peritoneum is repaired in male patients who undergo laparoscopic APR, there have been no more cases of intestinal obstruction. A previous study has reported much higher rates of anastomotic leakage in patients undergoing laparoscopic resection for rectal cancer (14) compared with those reported in our study (four patients; 2.6\%). Although intracorporeal rectal transection and anastomosis require great skill in patients undergoing laparoscopic low anterior resection, our group has completely standardized this technique in the Osaka Medical College Hospital, which has led to shorter operating times, low blood loss and a very low rate of conversion (13).

A previous study reported preoperative irradiation to be a risk factor for the development of surgical site infection (14). The wound infections occurring in the CRT group in our study included perineal wound infections that followed abdominoperineal resection, suggesting that a high rate of wound infection may not be a result of preoperative CRT, but rather is associated with differences in operative procedures among the different studies (15).

Through the use of several randomized controlled trials (16), surgeons in western countries have made preoperative CRT a standard therapeutic approach for the treatment of LARC. Surgeons in Japan, however, treat low rectal cancer with extended pelvic lymphadenectomy using TME. Retrospective Japanese studies have shown that this procedure decreases local recurrence while prolonging survival (17-19). Recently, several studies have compared CRT, the standard in western countries, with extended pelvic lymphadenectomy, the standard in Japan $(20,21)$. Despite a number of issues associated with these studies, a great step towards the future improvement of the treatment of low rectal cancer has been made by objectively comparing these two standards.

Several studies have demonstrated that preoperative CRT leads to an improvement in locoregional disease control, although they did not demonstrate that preoperative CRT leads to improved OS and RFS $(1-4,16,22)$. The results of the present study and those other recently performed studies are, therefore, similar. Our hypothesis is that adjuvant chemotherapy is likely to be of great importance in improving OS and RFS rates in the future.

In conclusion, although the present data was retrospectively collected, our results indicate that laparoscopic surgery with 
preoperative CRT appears to be safe and feasible, and may reduce local recurrence. However, future prospective studies and randomized controlled trials are required to clarify the benefits of preoperative CRT prior to laparoscopic surgery.

\section{References}

1. Kapiteijn E, Marijnen CA, Nagtegaal ID, Putter H, Steup WH, Wiggers T, Rutten HJ, Pahlman L, Glimelius B, van Krieken $\mathrm{JH}$, et al: Preoperative radiotherapy combined with total mesorectal excision for resectable rectal cancer. N Engl J Med 345: 638-646, 2001.

2. Gérard JP, Conroy T, Bonnetain F, Bouché O, Chapet O, Closon-Dejardin MT, Untereiner M, Leduc B, Francois E, Maurel J, et al: Preoperative radiotherapy with or without concurrent fluorouracil and lecovorin in T3-4 rectal cancers: Results of FFCD 9203. J Clin Oncol 24: 4620-4625, 2006.

3. Krook JE, Moertel CG, Gunderson LL, Wieand HS, Collins RT, Beart RW, Kubista TP, Poon MA, Meyers WC and Mailliard JA: Effective surgical adjuvant therapy for high-risk rectal carcinoma. N Engl J Med. 324 709-715, 1991.

4. Sauer R, Becker H, Hohenberger W, Rödel C, Wittekind C, Fietkau R, Martus P, Tschmelitsch J, Hager E, Hess CF, et al: Preoperative versus postoperative chemoradiation for rectal cancer. N Engl J Med 351: 1731-1740, 2004.

5. Akiyoshi T, Kuroyanagi H, Oya M, Konishi T, Fukuda M, Fujimoto Y, Ueno M, Yamaguchi T and Muto T: Safety of laparoscopic total mesorectal excision for low rectal cancer with preoperative chemoradiation therapy. J Gastrointest Surg 13: 521-525, 2009

6. Japanese Society for Cancer of the Colon and Rectum: Japanese classification of colorectal carcinoma. 2nd English edition. Kanehara \& Co., Ltd., Tokyo, 2009.

7. Clinical Outcomes of Surgical Therapy Study Group: A comparison of laparoscopically assisted and open colectomy for colon cancer. N Engl J Med 350: 2050-2059, 2004.

8. Guillou PJ, Quirke P, Thorpe H, Walker J, Jayne DG, Smith AM, Heath RM and Brown JM; MRCCLASICC trial group: Short-term endpoints of conventional versus laparoscopic-assisted surgery in patients with colorectal cancer (MRC CLASICC trial): Multicentre, randomized controlled trial. Lancet 365: 1718-1726, 2005.

9. Tjandra JJ, Chan MK and Yeh CH: Laparoscopic- vs. hand-assisted ultralow anterior resection: A prospective study. Dis Colon Rectum 51: 26-31, 2008.

10. Yamamoto M, Okuda J, Tanaka K, Kondo K, Tanigawa N and Uchiyama K: Clinical outcomes of laparoscopic surgery for advanced transverse and descending colon cancer: A single-center experience. Surg Endosc 26: 1566-1572, 2012.
11. Hayashi M, Komeda K, Inoue Y, Shimizu T, Asakuma M, Hirokawa F, Okuda J, Tanaka K, Kondo K and Tanigawa N: Simultaneous laparoscopic resection of colorectal cancer and synchronous metastatic liver tumor. Int Surg 96: 74-81, 2011.

12. Kayano H, Okuda J, Tanaka $\mathrm{K}$, Kondo $\mathrm{K}$ and Tanigawa $\mathrm{N}$ : Evaluation of the learning curve in laparoscopic low anterior resection for rectal cancer. Surg Endosc 25: 2972-2979, 2011.

13. Okuda J, Tanaka K, Kondo K, Asai K, Kayano H, Yamamoto M and Tanigawa N: Safe anastomosis in laparoscopic low anterior resection for rectal cancer. Asian J Endosc Surg 4: 68-72, 2011.

14. Bärlehner E, Benhidjeb T, Anders S and Schicke B: Laparoscopic resection for rectal cancer: Outcomes in 194 patients and review of the literature. Surg Endosc 19: 757-766, 2005.

15. Konishi T, Watanabe T, Kishimoto J and Nagawa H: Elective colon and rectal surgery differ in risk factors for wound infection: Results of prospective surveillance. Ann Surg 244: 758-763, 2006.

16. Bosset JF, Collette L, Calais G, Mineur L, Maingon P, Radosevic-Jelic L, Daban A, Bardet E, Beny A and Ollier JC; EORTC Radiotherapy Group Trial 22921: Chemotherapy with preoperative radiotherapy in rectal cancer. N Engl J Med 355: 1114-1123, 2006.

17. Koyama Y, Moriya Y and Hojo K: Effects of extended systematic lymphadenectomy for adenocarcinoma of the rectum-significant improvement of survival rate and decrease of local recurrence. Jpn J Clin Oncol 14: 623-632, 1984.

18. Moriya Y, Sugihara K, Akasu T and Fujita S: Importance of extended lymphadenectomy with lateral node dissection for advanced lower rectal cancer. World J Surg 21: 728-732, 1997.

19. Sugihara K, Moriya Y, Akasu T and Fujita S: Pelvic autonomic nerve preservation for patients with rectal carcinoma. Oncologic and functional outcome. Cancer 78: 1871-1880, 1996.

20. Kim JC, Takahashi K, Yu CS, Kim HC, Kim TW, Ryu MH, Kim JH and Mori T: Comparative outcomes between chemoradiation therapy and lateral pelvic lymph node dissection following total mesorectal excision in rectal cancer. Ann Surg 246: 754-762, 2007.

21. Nagawa H, Muto T, Sunouchi K, Higuchi Y, Tsurita G, Watanabe T and Sawada T: Randomized, controlled trial of lateral node dissection vs. nerve-preserving resection in patients with rectal cancer after preoperative radiotherapy. Dis Colon Rectum 44: 1274-1280, 2001.

22. Colorectal Cancer Collaborative Group: Adjuvant radiotherapy for rectal cancer: A systematic overview of 8,507 patients from 22 randomised trials. Lancet 358: 1291-1304, 2001. 